EPJ Web of Conferences 59, 17020 (2013)

DOI: $10.1051 /$ epjconf/20135917020

(C) Owned by the authors, published by EDP Sciences, 2013

\title{
Theoretical study of ultrarelativistic laser-electron interaction with radiation reaction
}

\author{
K. Seto ${ }^{1, a}$, H. Nagatomo ${ }^{1}$, J. Koga ${ }^{2}$, T. Taguchi ${ }^{3}$ and K. Mima ${ }^{1}$ \\ 1 Institute of Laser Engineering, Osaka Univ., Suita, Osaka, Japan \\ 2 Adv. Photon Research Center, Kansai Research Establishment, Japan Atomic Research \\ Institute, Kizu, Kyoto, Japan \\ ${ }^{3}$ Department of Electrical Engineering, Setsunan Univ., Neyagawa, Osaka, Japan
}

\begin{abstract}
When the laser intensity becomes higher than $10^{22} \mathrm{~W} / \mathrm{cm}^{2}$, the motion of an electron becomes relativistic, and emits large amounts of radiation. This radiation energy loss transferred to the kinetic energy loss of the electron, is treated as an external force, the "radiation reaction force". We show the new equation of motion including this radiation reaction and the simulation method, as well as results of single electron system or dual electrons system with Liénard-Wiechert field interaction.
\end{abstract}

\section{INTRODUCTION}

With the progress of ultra-short pulse laser technologies, peak intensity will reach $10^{22} \mathrm{~W} / \mathrm{cm}^{2}[1]$. We can classify the physical regime by the laser intensity in laser-electron interactions. With the intensity of $10^{18} \mathrm{~W} / \mathrm{cm}^{2}$, the electron becomes relativistic since the laser drives it to the speed of light. This is the region where we need to consider the ponderomotive force effect. As the intensity goes up to $10^{22} \mathrm{~W} / \mathrm{cm}^{2}$, it is predicted the "radiation reaction" effect appears [2], which is our main interest in this work. The electron loses the most of its inertia by its radiation. Therefore, the motion of electron changes from the case without the radiation. We can consider there is a new external force depending on the radiation. This effect is called the "radiation reaction" and this new force is the "radiation reaction force". When we need to consider the radiation reaction, this effect has to be formulated as the radiation reaction force, like external forces.

The original research into radiation reaction was related to the classical model of the electron by H. A. Lorentz in 1916 [3]. He considered the electron without the quantum theory. The summary of his model is as follows: The charge of the electron distributes on the surface of the sphere with classical electron radius $r_{\text {classic }}=\mathrm{O}\left(10^{-15} \mathrm{~m}\right)$. One of the small charged elements interacts with other elements through the Liénard-Wiechert electromagnetic field. When the electron moves, the self-interaction force (not the external force) works on the electron due to the directivity of the Liénard-Wiechert field. This self-interaction is the radiation reaction. The equation of motion with the radiation reaction in the nonrelativistic regime by Lorentz is called the Lorentz-Abraham (L-A) equation,

$$
m \dot{\mathbf{v}}=\mathbf{F}_{\mathrm{ex}}+m_{0} \tau_{0} \ddot{\mathbf{v}} .
$$

\footnotetext{
ae-mail: seto-k@ile.osaka-u.ac.jp
}

This is an Open Access article distributed under the terms of the Creative Commons Attribution License 2.0, which permits unrestricted use, distribution, and reproduction in any medium, provided the original work is properly cited. 
Here, $\tau_{0}=e^{2} / 6 \pi \varepsilon_{0} m_{0} c^{3}$ and $c$ is the speed of light. P. A. M. Dirac suggested a relativistic equation of L-A's with the Lorentz metric $g$, the signature of this is (+- - ) [4].

$$
m_{0} \frac{d w^{\mu}}{d \tau}=Q F_{\text {Laser }}^{\mu \nu} w_{v}+\left[m_{0} \tau_{0} \frac{d^{2} w^{\mu}}{d \tau^{2}}+\frac{m_{0} \tau_{0}}{c^{2}} g\left(\frac{d w}{d \tau}, \frac{d w}{d \tau}\right) w^{\mu}\right]
$$

where $\tau$ is the proper time, $w$ is the 4-velocity defined as $w \equiv \gamma(c, \mathbf{v})$ and $m_{0}$ is the rest mass of the electron. This Lorentz-Abraham-Dirac (LAD) equation and L-A equation have a significant defect in the energy exponential divergence called the run-away. Approximate methods have been suggested by Landau-Lifshitz [5] and Ford-O'Cornnell [6], but this method sacrifices the important physical rule of relativistic covariance. Rohrlich [7], Caldirola [8] and Sokolov [9] suggested other methods, but we need to wait to decide which is correct in nature. For this, all ideas which we can consider should be proposed. In this work, a new equation of motion with the radiation reaction $[10,11]$ is derived. In Section 2, we will show the outline of the derivation in the single electron system. Moreover, we will show the calculated result of the single and the dual electron system in Section 3 and Section 4.

\section{E.O. M. WITH RADIATION REACTION IN SINGLE ELECTRON SYSTEM}

The run-away problem in Eq. (2) depends on the second order derivative term, named the "Schott term". If every spacetime element includes this term, run-away happens. From another point of view, when one element does not have the Schott term, then the solution does not have an infinity. Therefore, we assume the time element does not have it $[10,11]$.

$$
m_{0} \frac{d w^{0}}{d \tau}=Q F_{\text {Laser }}{ }^{0 v} w_{v}+\frac{m_{0} \tau_{0}}{c^{2}} g\left(\frac{d w}{d \tau}, \frac{d w}{d \tau}\right) w^{0} .
$$

Here, the second term in the RHS means radiation energy loss,

$$
\frac{d W}{d t}=-m_{0} \tau_{0} g\left(\frac{d w}{d \tau}, \frac{d w}{d \tau}\right)=m_{0} c^{2} \tau_{0} \frac{\dot{\boldsymbol{\beta}}^{2}-(\boldsymbol{\beta} \times \dot{\boldsymbol{\beta}})^{2}}{\left(1-\boldsymbol{\beta}^{2}\right)^{3}} .
$$

Here, $\boldsymbol{\beta}=\mathbf{v} / c$. Using Eq. (3) with $g\left(w, f_{\text {reaction }}\right)=0$, the relativistic relation of the covariance,

$$
\begin{gathered}
m_{0} \frac{d w^{\mu}}{d \tau}=Q F_{\text {laser }}{ }^{\mu \nu} w_{\nu}+f_{\text {reaction }}{ }^{\mu} \\
f_{\text {reaction }}=m_{0} \tau_{0} \frac{d^{2} w^{\mu}}{d \tau^{2}}+\frac{m_{0} \tau_{0}}{c^{2}} g\left(\frac{d w}{d \tau}, \frac{d w}{d \tau}\right) w^{\mu}-m_{0} \tau_{0} \frac{w^{0} w^{\mu}-c^{2} \delta_{0}^{\mu}}{\left(w^{0}\right)^{2}-c^{2}} \frac{d^{2} w^{0}}{d \tau^{2}} .
\end{gathered}
$$

This equation is satisfied with the strict covariant form. (The detail of this derivation is in Section 2 in $[10,11])$. In Eq. (5) with Eq. (6), the radiation reaction effect is contained as the 4-force, we call it the "radiation reaction force". We will show the simulation result of Eq. (5) with Eq. (6), the single electron system in Section 3 and the dual electrons system in Section 4.

\section{SIMULATION RESULT OF A SINGLE ELECTRON SYSTEM}

In this section, we will show the simulation result of the laser-electron counter propagating case where the reaction force is larger than the laser's Lorentz force [10]. The Gaussian and linear polarization laser pulse has an intensity of $5 \times 10^{22} \mathrm{~W} / \mathrm{cm}^{2}$, a wavelength of $1 \mu \mathrm{m}$ and the pulse width is $20 \mathrm{fs}$. An electron is $200 \times \lambda_{\text {laser }}$ from the peak of the laser pulse. The calculation method is a simple leap-frog method, where the second derivatives in Eq. (6) are described by the two past time steps and present time step. The time step is defined by $\Delta \tau=10^{-4} / \gamma_{\text {initial }} \times$ (Laser period). The simulation result is shown in Fig. 1 . In (a) and (b), the blue line is the case with the radiation reaction and the red line is without the radiation 


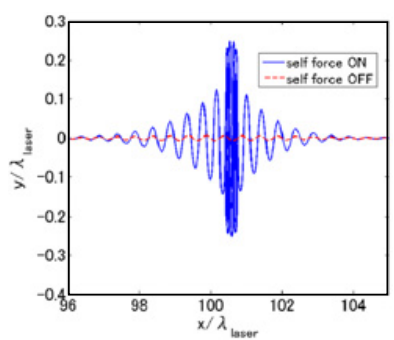

(a) Trajectory

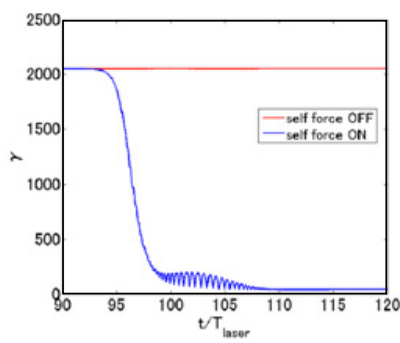

(b) Energy change

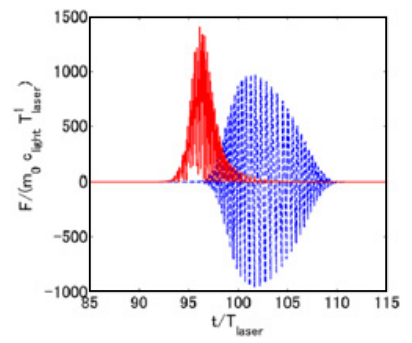

(c) Reaction force

Figure 1. The characteristic of the electron's motion.

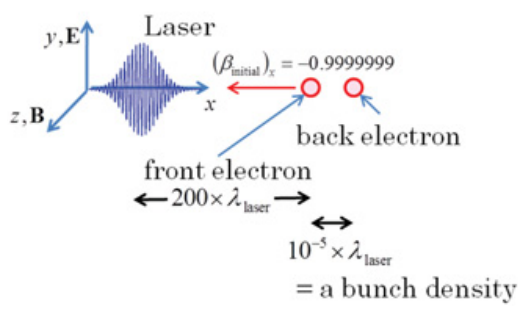

Figure 2. Configuration of the dual electron system.

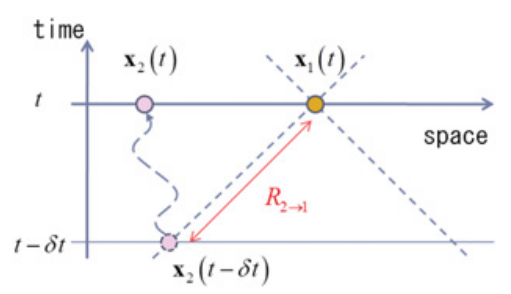

Figure 3. Calculation of the retarded field.

reaction. In the case without the radiation reaction, the electron moves with an initially large inertia and does not lose its energy. But with the radiation reaction, the electron loses its inertia by the radiation. And this radiation reaction is the red line in Fig. 1(c). This is a plot of the x component of Eq. (6). The blue one is the $\mathrm{x}$ direction of the Lorentz force. This figure shows that the radiation reaction force can become larger than the laser-Lorentz force. This mechanism is the reason why the motion of the electron is deformed, and the reason why the radiation reaction should be considered. In fact, the most effective term in Eq. (6) is the second term, the direct radiation $[10,11]$. This is the same in the L-L method. We discussed theoretically this point in [7]. It is shown that our new Eq. (5) with Eq. (6) and the simulation result contain the result of the L-L equation.

\section{SIMULATION RESULT OF DUAL ELECTRON SYSTEM}

In Section 3, we show Eq. (5) with Eq. (6) are suitable for the description of the electron's motion with the radiation reaction. Next, we proceed to two electron system. The configuration of the simulation is given in Fig. 2. The laser's characteristics are the same as those of the single electron system. We take the distance of $10^{-5} \times \lambda$ laser between the two electrons. In this dual electron system, we need to consider the electromagnetic field interaction between each particle. This field is described by the Liénard-Wiechert field as the solution of Maxwell's equations [3-5]. The electric field which is emitted from Charge 2 and then is captured by Charge 1, is as follows.

$$
\mathbf{E}_{\mathrm{L}-\mathrm{W}}^{2 \rightarrow 1}(\mathbf{x}, t)=\frac{Q_{2}}{4 \pi \varepsilon_{0} c} \frac{\mathbf{n}_{2 \rightarrow 1} \times\left(\mathbf{n}_{2 \rightarrow 1}-\boldsymbol{\beta}_{2}\right) \times \dot{\boldsymbol{\beta}}_{2}}{\left(1-\mathbf{n}_{2 \rightarrow 1} \cdot \boldsymbol{\beta}_{2}\right)^{3} R_{2 \rightarrow 1}}+\frac{Q_{2}}{4 \pi \varepsilon_{0}} \frac{\left(1-\boldsymbol{\beta}_{2}^{2}\right)\left(\mathbf{n}_{2 \rightarrow 1}-\boldsymbol{\beta}_{2}\right)}{\left(1-\mathbf{n}_{2 \rightarrow 1} \cdot \boldsymbol{\beta}_{2}\right)^{3} R_{2 \rightarrow 1}{ }^{2}} .
$$

The magnetic field is described by $\mathbf{B}_{\mathrm{L}-\mathrm{W}}^{2 \rightarrow 1}(\mathbf{x}, t)=c^{-1} \mathbf{n}_{2 \rightarrow 1} \times \mathbf{E}_{\mathrm{L}-\mathrm{W}}^{2 \rightarrow 1}(\mathbf{x}, t)$. Where the vector $\mathbf{n}_{2 \rightarrow 1}$ is the unit vector, $\mathbf{n}_{2 \rightarrow 1}=\left[\mathbf{x}_{1}(t)-\mathbf{x}_{2}(t-\delta t)\right] /\left|\mathbf{x}_{1}(t)-\mathbf{x}_{2}(t-\delta t)\right|$. The Liénard-Wiechert field is an electromagnetic field, the interaction is treated as the Lorentz force of ${ }_{1} Q F_{\mathrm{L}-\mathrm{W}}^{2 \rightarrow 1 \mu \nu} w_{\nu}$. Therefore, the 


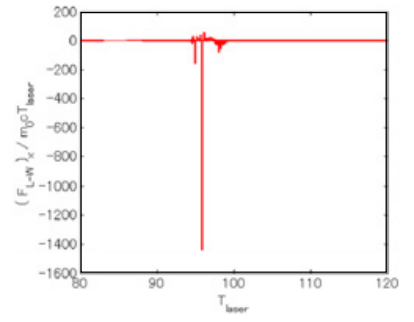

Figure 4. Interaction force which kicks the front electron.

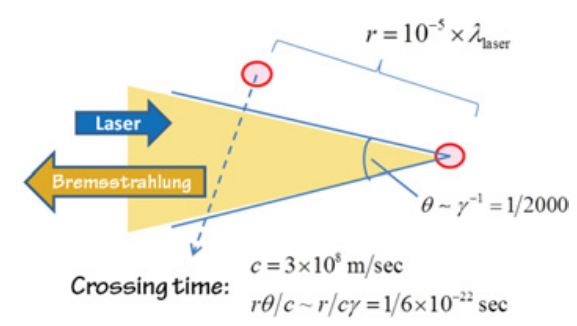

Figure 5. Interaction by the radiation from the back electron.

equation of motion on Charge 1 is

$$
{ }_{1} m_{0} \frac{d}{d \tau}{ }_{1} w^{\mu}={ }_{1} Q F_{\text {laser }}^{\mu \nu}{ }_{1} w_{\nu}+{ }_{1} Q F_{\mathrm{L}-\mathrm{W}}^{2 \rightarrow 1 \mu \nu}{ }_{1} w_{\nu}+{ }_{1} f_{\text {reaction }}{ }^{\mu} .
$$

Where the values of $1 \bullet$ mean the parameters of Charge 1. Applying this to Eq. (8) on the dual electron system, the simulation result of ${ }_{1} Q F_{\mathrm{L}-\mathrm{W}}^{2 \rightarrow 11 v} w_{\nu}$ (x direction kicking) is shown in Fig. 4. It has strong interaction peak in a very small time interval. Figure 6 is the reason why the interaction becomes Fig. 4. The motion of the electrons is almost the same as Fig. 1(a) with the interaction. Therefore, the radiation profile becomes Fig. 1(b). Here, the emission is in the $\mathrm{x}$ direction with a very narrow emission angle, the forward-irradiated electron interacts a very short time when the electron intersects with radiation. The effect for an electron motion is decided by the power of the Liénard-Wiechert fields. This means the Liénard-Wiechert force acts in a very small interval, and the total motion of each electron is almost the same as in the case without the interaction. If we take a longer distance as the initial electron setup, the irradiating area will be expanded. Stronger influences are expected than in this comoving case with the very short distance.

\section{CONCLUSION}

This paper introduces our new model which considers the radiation reaction and some simulation results produced by this model. Our Eq. (5) with Eq. (6) maintains relativistic covariance and involves the results of the major method of the L-L equation. Therefore, this equation is more general than the L-L method. Moreover, we have succeeded with two electrons using the Liénard-Wiechert interaction. Since the directivity of the irradiated radiation is too narrow, the strong interacting interval is very short. Therefore, the Liénard-Wiechert interaction is smaller than other forces, the external laser's Lorentz force and the radiation reaction.

\section{References}

[1] V. Yanovsky et al., Opt. Express 16, 2109 (2008)

[2] J. Koga, Phys. Rev. E 70, 046502 (2004)

[3] H. A. Lorentz, The Theory of Electrons and Its Applications to the Phenomena of Light and Radiant Heat (Leipzig, New York, $2^{\text {nd }}$ Ed., 1916)

[4] P. A. M. Dirac, Proc. Roy Soc. A 167, 148 (1938)

[5] L. D. Landau and E. M. Lifshitz, The Classical theory of fields (Pergamon, New York, 1994)

[6] G. W. Ford and R. F. O'Connell, Phys. Lett. A 174, 182 (1993)

[7] F. Rohrlich, Am. J. Phys. 65 (11), (1997)

[8] P. Caldirola, Nuovo Cimento 3, Suppl. 2, 297 (1956)

[9] I. V. Sokolov, J. Exp. Theo. Phys. 109, No.2 (2009)

[10] K. Seto, H. Nagatomo and K. Mima,Plasma Fusion Res. 6, 2404099 (2011)

[11] K. Seto, H. Nagatomo, J. Koga and K. Mima. Phys. Plasmas 18, 123101 (2011) 